\title{
NUMERICAL SIMULATIONS OF 3D COMPRESSIBLE CONVECTION
}

\author{
HERBERT J. MUTHSAM \\ JOHANN ZÖCHLING \\ Institute of Mathematics \\ University of Vienna \\ Boltzmanngasse 5 \\ A-1090 Vienna/Austria \\ e-mail: A8131DAA@AWIUNI11.BITNET
}

\begin{abstract}
Numerical models of a convection zone embedded between two stable layers have been calculated; one is shortly presented and in particular a few items concerning the lower overshoot region are discussed.
\end{abstract}

\section{INTRODUCTION}

As one of the large, still in many a respect unsettled questions of stellar physics, the study of compressible convection has, in recent years, attracted the attention of a growing number of research groups using the approach of numerical modelling on the basis of the (more or less full) equations of hydrodynamics, with appropriate extension included. See e.g. Cattaneo et al. (1991), Chan and Sofia (1989), Hossain and Mullan (1991), Fox et al. (1991) for recent work.

We contribute here to such efforts and discuss a few items which have shown themselves when studying a $3 \mathrm{D}$ model of a convection zone which is bounded above and below by stable zones (thus essentially eliminating the effect of more or less artificial boundary conditions which arise when the convection zone is bounded by the margin of the computational domain). For 2D work see Hurlburt et al. (1986).

\section{EQUATIONS}

We have developed a numerical code ASCIC 90 (A Stellar Convection Investigation Code for the 90's) which allows to integrate in time the pertinent equations. In its present form it is based on central differencing on 
a staggered mesh and a leapfrog method in time. We are presently extending it to an ENO-scheme of high order, see Lafon and Osher (1991).

The equations are the compressible Navier-Stokes equations inside a box with a heat conduction term (= radiative transfer in the diffusion approximation) added. The boundary conditions are as in Hurlburt et al. (1986).

In order to get insight to the basic physics, we have adopted simple microphysics; we take as starting configuration polytropic layers situated atop of each other with parameters matched thus that consistency (same flux everywhere, etc.) is guaranteed. The coefficients of radiative conductivity and viscosity are held fixed at their initial values at each depth. The equation of state is that of a perfect gas.

Although our code would allow for such, subgrid modelling is not used in the model described in this note.

\section{SHORT DISCUSSION OF A MODEL}

We discuss model $003 \mathrm{~F}$ in our designation. It is calculated on a $72 * 50 * 50$ grid (72 depth points) and has an aspect ratio of $1: 2: 2$. It is characterized by the following data:

\begin{tabular}{|l|l|l|c|l|}
\hline depth & $\beta$ & $\rho / \rho_{\text {top }}$ & $p / p_{\text {top }}$ & $T / T_{\text {top }}$ \\
\hline 0.0 & 0.3 & 1. & 1. & 1. \\
0.2 & 0.8 & 3.11 & 4.34 & 1.39 \\
0.3 & 1.25 & 4.28 & 7.51 & 1.75 \\
0.55 & 1.25 & 7.12 & 20.5 & 2.90 \\
0.65 & 0.3 & 9.1 & 27.3 & 3.11 \\
1.0 & 0.3 & 19.8 & 69.0 & 3.47 \\
\hline
\end{tabular}

The total depth ranges from 0 to 1 . The values of $\rho$ etc. are given in units of their values at the top; they are naturally only valid for the hydrostatic starting configuration. $\beta$ is the temperature gradient in units of the adiabatic one. Thus, the main part of the convection zone is between depths 0.3-0.55. Its Rayleigh Number is $10^{6}$.

We show in fig. 1 various fluxes. Of special importance are the radiative flux (FRAD) and convective flux FCONV. As is seen, the lower stable region has not yet quite converged to the nominal flux $(=-1)$. Of special interest is that at the bottom of the convection zone more than the nominal flux is transported by radiation whereas convection transports there heat downwards. We see this effect also in many other models.

We also hint at fig. 2. Here, correlation coefficients of pairs of quantities are evaluated at a horizontal plane and plotted as a function of depth. XC DG $T$ (X-Correlation of Density of Gas with Temperature) shows a rather dull behaviour, being close to -1 over most of the depth-range. Thus, as expected, denser material is, on the average, cooler. 


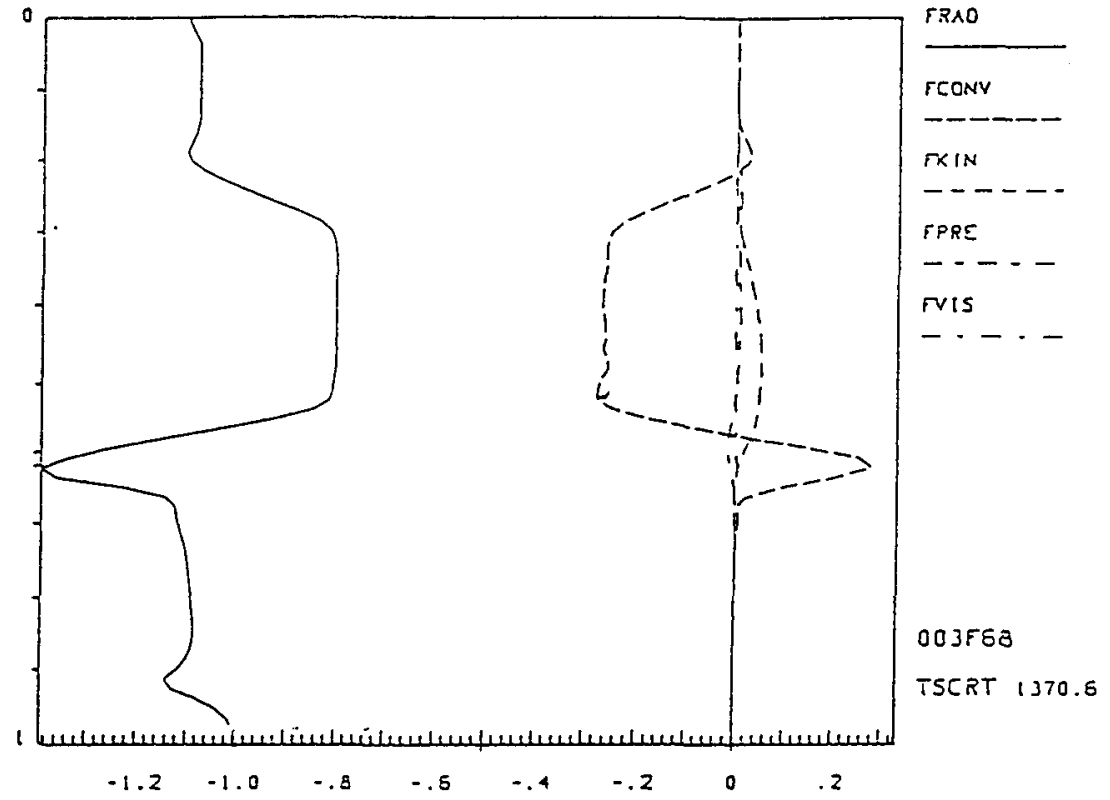

Fig. 1. Radiative and convective flux plus some fuxes of lesser importance plotied in dependenct of depth (ordinate). The nomial Aux is -1 .

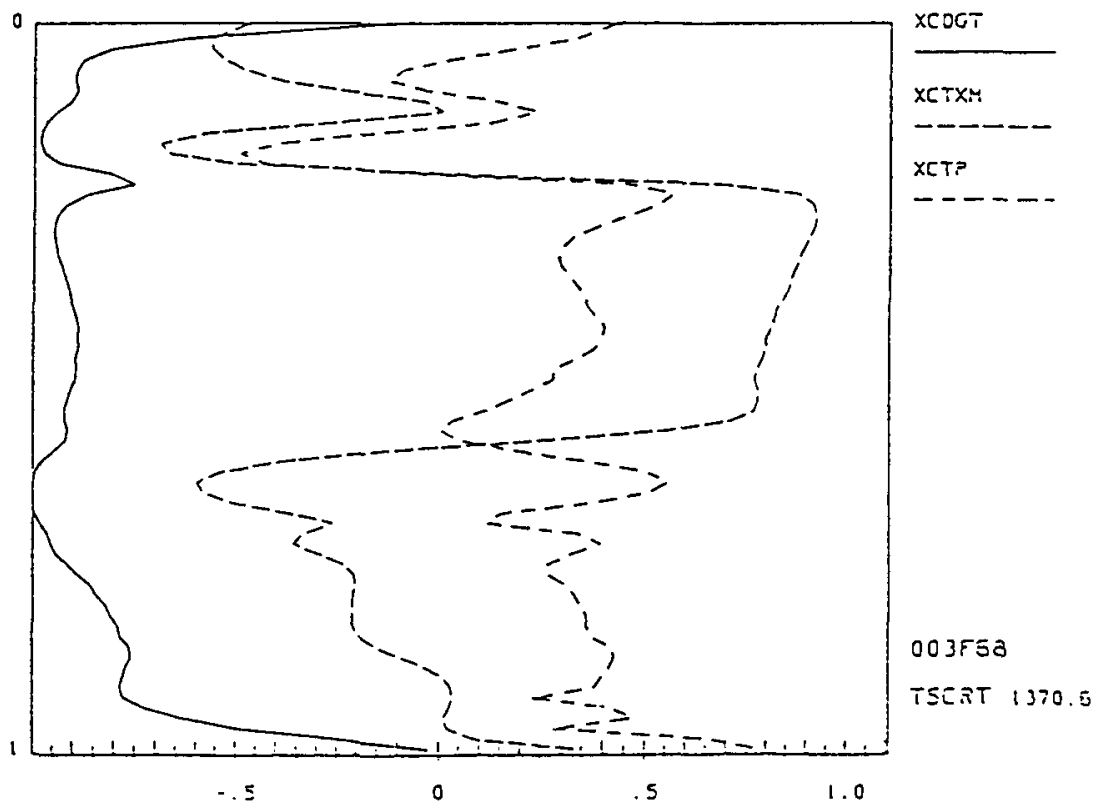

Fig. 2. Correlations of physical quantities over horizontal planes in dependency of depth (ordinate). For explanation see text. 
XC T XM (X-Correlation of Temperature fluctuations about horizontal average with $\mathrm{X}$-Momentum [vertical]) is much more interesting; in particular, it being negative at the lower boundary of the convection zone again tells that there heat is transported downwards by convection. This quantity and also XC T P ( $P=$ pressure) show considerable depth dependence. Effects that can be discerned by these and other data will be reported elsewhere, Muthsam, (1992).

\section{ACKNOWLEDGEMENT}

Calculations were performed at IBM 3090-400 VF, IBM 9021-720 computers and at an IBM RS 6000-550 workstation in the framework of EASI, European Academic Supercomputer Initiative, at the Computing Center, University of Vienna. Early stages of the work were granted by the Fonds zur Förderung der wiss. Forschung, project 4122.

\section{REFERENCES}

Cattaneo, F. et al. 1991, Ap. J., $\underline{370}, 282$.

Chan, K.L. and Sofia, S. 1989, Ap. J., 336, 1022.

Fox, P.A., Theobald, M.L. and Sofia, S. 1991, Ap. J., $\underline{383}, 860$.

Hossain, M. and Mullan, D.J., 1991, Ap. J., $\underline{380}, 631$.

Hurlburt, N.E. et al. 1986, Ap. J., $\underline{311}, 563$.

Lafon, F. and Osher, S. 1991, Journal of Computational Physics, 96, 110.

Muthsam, H. 1992, in preparation. 